\title{
Trichinella spiralis: RNAi-mediated silencing of serine protease results in reduction of intrusion, development and fecundity
}

\author{
Yang, D.Q., Zeng, J., Sun, X.Y., Yue, X., Hu, C.X., Jiang, P., Liu, R.D., Cui, J.* and Wang, Z.Q.* \\ Department of Parasitology, Medical College; Zhengzhou University, 40 Daxue Road, Zhengzhou 450052, \\ People's Republic of China \\ *Corresponding author e-mails: wangzq@zzu.edu.cn, wangzq2015@126.com (Wang, Z.Q.) or \\ cuij@zzu.edu.cn (Cui, J.)
}

Received 3 June 2020; received in revised form 13 August 2020; accepted 13 August 2020

\begin{abstract}
In previous studies, a Trichinella spiralis serine protease (TsSP) was identified in excretion/secretion (ES) products from intestinal infective L1 larvae (IIL1) using immunoproteomics. The complete cDNA sequence of TsSP gene was $1372 \mathrm{bp}$, which encoded 429 amino acids with $47.55 \mathrm{kDa}$. The TsSP was transcribed and expressed at all T. spiralis life cycle phases, as well as mainly located at the cuticle and stichosome of the parasitic nematode. Recombinant TsSP bind to intestinal epithelial cells (IEC) and promoted larva invasion, however, its exact function in invasion, development and reproduction are still unknown. The aim of this study was to confirm the biological function of TsSP during T. spiralis invasion and growth using RNA interference (RNAi) technology. The results showed that on 1 day after electroporation using $2.5 \mu \mathrm{M}$ siRNA156, TsSP mRNA and protein expression of muscle larvae (ML) was suppressed by 48.35 and 59.98\%, respectively. Meanwhile, silencing of TsSP gene by RNAi resulted in a $61.38 \%$ decrease of serine protease activity of ML ES proteins, and a significant reduction of the in vitro and in vivo invasive capacity of IIL1 to intrude into the IEC monolayer and intestinal mucosa. When mice were infected with siRNA 156-transfected larvae, adult worm and muscle larva burdens were decreased by 58.85 and $60.48 \%$, respectively. Moreover, intestinal worm growth and female fecundity were evidently inhibited after TsSP gene was knockdown, it was demonstrated that intestinal adults became smaller and the in vitro newborn larval yield of females obviously declined compared with the control siRNA group. The results indicated that knockdown of TsSP gene by RNAi significantly reduced the TsSP expression and enzymatic activity, impaired larvae intrusion and growth, and lowered the female reproductive capacity, further verified that TsSP might participate in diverse processes of $T$. spiralis life cycle, it will be a new prospective candidate molecular target of anti-Trichinella vaccines.
\end{abstract}

\section{INTRODUCTION}

Trichinella spiralis (Owen, 1835) is a major enzootic nematode distributed in more than 150 mammals around the world (Pozio, 2007). Humans acquire trichinellosis by consuming raw or semi-raw meat or meat products infected with Trichinella muscle larvae (ML) (Cui et al., 2011). T. spiralis not only harms human health, but also poses a serious threat to meat food safety (Cui \& Wang, 2011; Cui et al., 2013). At the same time, T. spiralis is ranked as the seventh more important foodborne parasitic disease in the world (FAO/WHO, 2014). It is an important public health issue on Trichinella infection in domestic pigs and a tremendous risk for pork and pork product safety (Rostami, 2017). Due to the widespread distribution of Trichinella animal hosts and the lack of practical prophylactic vaccines (Liu et al., 2015a; Qi et al., 2018a), it is therefore difficult to control Trichinella infection (Jiang et al., 2016; Bai et al., 2017). These 
problems prompted the identification of T. spiralis invasion-associated proteins as molecular targets for anti-Trichinella vaccines (Wang et al., 2012b; Song et al., 2018a; Xu et al., 2018).

Serine protease is an important family of proteases, which play an important role in host cell and tissue invasion, worm development, anticoagulation and immune evasion (Yang et al., 2015). There are more and more researches on serine proteases of parasitic nematodes, mainly including Trichuris muris, Ascaris lumbricoides, Brugia malayi, and T. spiralis (Cui et al., 2015). Some serine proteases have been identified from different $T$. spiralis life cycle stages (Robinson et al., 2005; Wang et al., 2013a, 2013b). Furthermore, serine protease expression at intestinal infective L1 larvae (IIL1) was higher than the muscle larval stage (Ren et al., 2013; Liu et al., 2013, 2015b). Antibodies against T. spiralis serine proteases (TspSP1 and TspSP1.2) inhibited the enterocyte invasion (Romaris et al., 2002; Wang et al., 2013c). Vaccination of mice with recombinat (rTsSP) protein or DNA vaccine exhibited a significant immune protection, as demonstrated by an obvious worm burden reduction of enteral adult worms (AW) and mucle larvae (ML) in vaccinated mice after challenge infection (Li et al., 2018; Ren et al., 2018). The results demostrated that serine proteases may extert a main act for $T$. spiralis invasion and development inside the host.

In our previous studies, a serine protease (TsSP, GenBank: ABY60762), which had been screened in excretion/secretion (ES) products from $T$. spiralis IIL1 and adults using immunoproteomics (Liu et al., 2016a, 2016b; Wang et al., 2017), was cloned, expressed and characterized in our laboratory (Sun et al., 2018b). The results showed that the complete cDNA sequence of TsSP gene was $1372 \mathrm{bp}$ and encoded 429 amino acids with $47.55 \mathrm{kDa}$. The TsSP was transcribed and expressed at all T. spiralis life cycle phases, it mainly located at the cuticle and stichosome of the parasitic nematode. The rTsSP could bind with intestinal epithelial cell (IEC) and promoted the larva invasion of IEC. Immunization with rTsSP elicited a significant immune response and protection effect, which hindered the worm development and decreased the female adult fecundity, resulted in a $71.1 \%$ adult reduction at 5 days post infection (dpi) and a 62.1\% ML reduction at $42 \mathrm{dpi}$ (Sun et al., 2018a; 2019b). Although the TsSP is expected to be a prospective molecular target of antiTrichinella vaccine, the exact role of TsSP in worm invasion, development and survival in host have not been well elucidated.

The aim of the present study was to confirm the biological function of TsSP during T. spiralis invasion, development and fecundity by using RNA interference (RNAi) technology. Three kinds of TsSPspecific small interfering RNA (siRNAs) were used to silence the expression of TsSP in T. spiralis ML. The TsSP enzyme activity and in vitro larval invasive capacity were observed after silencing TsSP. Moreover, the development, morphology and fecundity of siRNA-treated worms from the infected mice were also investigated.

\section{MATERIALS AND METHODS}

\section{Worm and mice}

The T. spiralis isolate (ISS534) was recovered from a naturally infected pig in Henan Province of China and kept by serial passage in $\mathrm{BALB} / \mathrm{c}$ mice in our laboratory (Wang et al., 2012a). BABL/c mice (female, 15-20 g) were obtained from the animal center of Zhengzhou University. Animal experiment was conducted according to the National Guidelines for Experimental Animal Welfare (Minister of Science and Technology, the People's Republic of China, 2006). Experiment protocols were approved by the Institutional Life Science Ethics Committee, Zhengzhou University (No. SCXK 2017-0001).

\section{SiRNA preparation}

Complete cDNA encoding TsSP was utilized to design the siRNA sequences by using siDirect version 2.0 (Naito et al., 2009). Synthesis of TsSP-specific $21 \mathrm{bp}$ siRNAs 
was done by Sangon Biotech (Shanghai, China). To select the better performance of TsSP-specific siRNA in silencing the TsSP mRNA expression and the time of transfection, three TsSP-specific siRNAs, SiRNA-156 (5'-UUUCUGAACACUAAUCUU GTT-3'), siRNA-171 (5'-GAAAAG CGGGUAA UAGAAUTT-3'), and siRNA-436 (5'-UACAU AUUCUUUAAG CAUCTT-3') were used in the present study. A control siRNA carrying a scrambled sequence (5'-AUCGGCUACC AAGUCAUACTT-3') was served as a control. The control siRNA was fluorescently labeled with FAM (Sangon Biotech, Shanghai, China) and used to assess the transfection efficiency. The specific siRNA and polyclonal antibodies of T. spiralis glutathione S-transferase (TsGST) was also prepared and used for control of the specificity (Wang et al., 2015; Yang et al., 2019).

\section{Delivery of siRNA into ML}

The ML were obtained via artificial digestion of experimentally infected mouse muscles at 35 dpi (Jiang et al., 2012). Following washes with electroporation buffer, 5000 fresh larvae were suspended in $100 \mu \mathrm{l}$ of electroporation buffer contained $1 \mu \mathrm{M}$ one siRNA of three TsSP-specific siRNAs (Zhang et al., 2016). The worms were transfected by siRNA through electroporation, and cultivated in RPMI 1640 culture medium at $37^{\circ} \mathrm{C}$ for 1-7 days (Chen et al., 2012).

\section{qPCR}

Total RNA from siRNA-transfected and control worms was extracted using Trizol reagent (Invitrogen), transcribed into the first-strand cDNA with PrimeScript RT reagent Kit (TaKaRa, Japan) (Ren et al., 2013). The TsSPI mRNA expression levels were ascertained using qPCR (Song et al., $2018 b$ ). The specific primers of qPCR amplifying TsSP gene were 5'-CAGAATTT CAGGAGGCTCTGTTG-3', and 5'-ACCACGT CCACCTTGATATGT-3'. A $\beta$-actin gene was used as a housekeeping gene control (Martinez et al., 2001; Sun et al., 2018a). Each sample has three repeats. The quantitative datum was analyzed by comparative Ct $\left(2^{-\Delta \Delta \mathrm{Ct}}\right)$ method (Liu et al., 2018).
Western blotting of TsSP protein expression in siRNA-transfected ML somatic proteins

Somatic soluble worm proteins of siRNAtreated ML were prepared as reported before (Wang et al., 2011; Li et al., 2015). Worm proteins (10 $\mathrm{\mu g} /$ lane) were separated using SDS-PAGE, transferred onto polyvinylidene difluoride (PVDF) membrane at $18 \mathrm{~V}$ for $35 \mathrm{~min}$ via semidry transferred cell (Bio-Rad) (Song et al., 2018c). The membrane was cut into strips, which were blocked for $2 \mathrm{~h}$ at $37{ }^{\circ} \mathrm{C}$ in TBST with $5 \%$ nonfat milk, and incubated for $1 \mathrm{~h}$ at $37^{\circ} \mathrm{C}$ with anti-TsSP antibodies (1:100) prepared in our previous study (Sun et al., 2019a). After washing, the strips were incubated with HRP-conjugated-anti-mouse IgG (1:10000; Sigma-Aldrich, USA). $\beta$-actin expression was also determined by using anti- $\beta$-Actin antibody (1:1000). The strips were stained with an enhanced chemiluminescent kit (CWBIO, Beijing, China) (Wang et al., 2014). The protein bands were analyzed with the AlphaView Software, and relative protein expression level was assessed based on densitometry (Sun et al., 2018b).

\section{Enzymatic activity assay of worm native} serine protease after TsSP gene silencing After being electroporated with $2.5 \mu \mathrm{M}$ siRNA 156 , the MLs were cultivated at $37^{\circ} \mathrm{C}$ in a $5 \% \mathrm{CO}_{2}$ for $24 \mathrm{~h}$. The parasites were removed from the medium by centrifugation $(100 \times \mathrm{g}$ for $5 \mathrm{~min})$. The ES proteins were collected after the cultured ML (Zhang et $a l .$, 2013). And then, $100 \mu \mathrm{l}$ of treated-ML ES proteins co-incubated with $200 \mu \mathrm{l}$ of $1 \mathrm{mg}$ $\mathrm{ml}^{-1}$ azocasein (Sigma-Aldrich, USA) at $37^{\circ} \mathrm{C}$ for $60 \mathrm{~min}$. Reactions were stopped by adding $10 \%$ trichloroacetic acid (TCA), the mixtures were left for $15 \mathrm{~min}$ and then centrifuged at $600 \mathrm{~g}$ for $10 \mathrm{~min}$, and finally, $1 \mathrm{M} \mathrm{NaOH}$ was added to $1 \mathrm{ml}$ of the supernatant. Absorbance at $440 \mathrm{~nm}$ was determined (Ramírez-Flores et al., 2019). Every group had three independent repeats.

\section{Zymography}

SDS-PAGE gel containing gelatin $\left(1 \mathrm{mg} \mathrm{ml}^{-1}\right)$ were used for zymography of $T$. spiralis ML somatic and ES proteins after larval 
transfection with siRNA 156 (LorenzoMorales et al., 2005). Briefly, $20 \mu \mathrm{l}$ of somatic or ES proteins were loaded onto an SDS-PAGE gel containing gelatin $\left(1 \mathrm{mg} \mathrm{ml}^{-1}\right)$. Following electrophoresis, SDS was removed by washing twice with $2.5 \%$ Triton solution, and the gel was incubated at $37^{\circ} \mathrm{C}$ for $36 \mathrm{~h}$ in $0.1 \mathrm{M}$ Tris base buffer solution $(\mathrm{pH}$ 8.0) containing $2 \mathrm{mM} \mathrm{NaN}$. Then the gel was put into Coomassie Brilliant Blue R-250 Decolorizer for $3 \mathrm{~h}$ and scanned. The experiment was repeated three times.

\section{The in vitro survival rate of siRNA-156 transfected ML}

The ML were treated with $2.5 \mu \mathrm{M}$ siRNA-156 and cultured in RPMI 1640 medium at $37^{\circ} \mathrm{C}$ and $5 \% \mathrm{CO}_{2}$ for $1-7$ days. The survival rates of siRNA-156 transfected ML were evaluated in light of larval morphology and motility by microscopy. The live worms are mobile and exhibit a wriggling movement, whereas the dead parasite is "C" shaped or straight and inactive (Liu et al., 2018). The result was presented as the percent of dead worms to all of the worms observed in each group.

\section{In vitro larval intrusion test}

To assess the silencing TsSP on in vitro larva intrusion of IEC, the ML were transfected with $2.5 \mu \mathrm{M}$ siRNA-156 and cultivated for 1 day. Then, the siRNA-treated ML were activated into the IIL1 larvae using 5\% mouse bile, and used in the intrusion test (ManWarren et al., 1997; Long et al., 2015). The IECs were cultured in a 6-well culture plate and the cell monolayer was covered with 100 IIL1 larvae in $2 \mathrm{ml}$ of DMEM semisolid medium (Qi et al., 2018b; Xu et al., 2018). After culture at $37^{\circ} \mathrm{C}$ for $2 \mathrm{~h}$, the IIL 1 that invaded into the IEC were examined and numbered under microscopy. The larvae that were motile and migrated within the IEC monolayer were taken as invaded larvae, whereas the larvae that was suspended in the culture medium and coiled were regarded as non-invaded larvae (McVay et al., 1998; Xu et al., 2020). Three repeats were performed to determine the percentage of the invaded larvae in each group (Ren et al., 2018).
The in vitro larval invasion of enteral epithelium of isolated intestine

The siRNA 156- treated ML were activated into the IIL1 using $5 \%$ mouse bile at $37^{\circ} \mathrm{C}$ for $2 \mathrm{~h}$, and used for enteral epithelium invasion test. The mouse small intestine was cut into 2-3 cm, two ends of intestinal segment were clamped and ligated to form an intestinal pouch, and each segment were perfused with 100 siRNA 156- treated IIL larvae. After being incubated in Tyrode's solution at $37^{\circ} \mathrm{C}$ for $2 \mathrm{~h}$, the intestine was cut off, compressed on grass side and examined by microscopy, the worms remaining in enteral lumen were assessed as non-invaded ones (Long et al., 2015; Yang et al., 2020). Three repeats were applied in each test.

\section{Assessment of infectivity and fecundity of siRNA-treated larvae}

To assess the infectivity, developmental capacity and fecundity of siRNA-treated larvae, 60 mice were equally divided into three groups (20 mice/group). Each mouse was orally administrated by gavage with 300 ML treated using siRNA-156, control siRNA or PBS. Intestinal adult worms (AW) were collected from 10 mice of each group at 6 dpi (Cui et al., 2013; Sun et al., 2015). The remaining 10 mice of each group were euthanized at $35 \mathrm{dpi}$, and the ML were obtained by artificial digestion of mouse carcasses as previously described (Jiang et $a l ., 2012)$. The parasite burden reduction was estimated by means of gut AW burden and larvae per gram (LPG) of muscles collected from siRNA-156 group relative to those from the PBS group (Cui et al., 2019). Furthermore, the reproductive capacity of adult females was determined according in the light of the in vitro NBL yield of each female in $72 \mathrm{~h}$ (Yang et al., 2019). The length of various stage worms from infected mice was assessed under microscopy.

\section{Statistical Analysis}

The data were analyzed by SPSS 22.0 software, and shown as means \pm standard deviation (SD). Differences of relative TsSP expression level, parasite burden and 
length, and NBL yield among various groups were analyzed with one-way ANOVA, $P<0.05$ was assessed as statistically significant difference.

\section{RESULTS}

\section{Transfection of siRNA into ML}

At $18 \mathrm{~h}$ following electroporation with control siRNA-labeled fluorescein, $91 \%$ of the ML showed fluorescence staining in different tissues under a fluorescent microscopy (Figure 1), suggested that siRNA has been delivered into the ML with electroporation.

\section{Decrease of TsSP mRNA expression in T. spiralis ML with siRNAs}

After being transfected with $1 \mu \mathrm{M}$ of siRNA 156 and siRNA 171 for $24 \mathrm{~h}$, the TsSP mRNA expression level in the ML electropporated by two siRNAs resulted in 50.54 and $36.36 \%$ reduction compared with those of the PBS group, respectively $(P<0.01)$. When the siRNA 436 and control siRNA were used, no evidently inhibition of TsSP mRNA expression was observed relative to the PBS group $(P>0.05$ (Figure $2 \mathrm{~A}$ ). While the ML were transfected with $1.5,2.0,2.5,3.0$ and 3.5 $\mu \mathrm{M}$ siRNA 156, TsSP mRNA expression reduced by $50.49,51.49,55.48,50.14$ and $48.47 \%$, respectively, compared with the PBS group $(F=12.261, P<0.0001)$ (Figure $2 \mathrm{~B})$.
On 1, 3, 5 and 7 days after being transfected by $2.5 \mu \mathrm{M}$ of siRNA 156 , the worm TsSP mRNA expression decreased by $48.35,36.68$, 35.01 and $21.75 \%$ respectively $(F=26.195$, $P=0.001)$. Nevertheless, the TsSP mRNA expression in control siRNA- treated ML group had no distinct decrease $(P>0.05)$ (Figure 2C).

\section{Decrease of worm TsSP protein expression following transfection with siRNA}

Western blotting results showed that larval TsSP protein expression was 54.55, 46.84 and $30.06 \%$ reduction after transfection using siRNA-156, siRNA-171 or siRNA-436, respectively. The siRNA-156 treated worms had a significant reduction relative to the control siRNA and PBS groups $(P<0.05)$ (Figure 3A). When various siRNA-156 concentrations $(1.5,2.0,2.5,3.0$, and $3.5 \mu \mathrm{M})$ were used, TsSP protein expression levels reduced by $49.62,44.38,58.63,51.91$ and $54.38 \%$, respectively. While $2.5 \mu \mathrm{M}$ siRNA156 was used for 1 day, it resulted in the highest inhibition of TsSP protein expression $(P<0.05)$ (Figure 3B). At 1, 3, 5 and 7 days after treatment with $2.5 \mu \mathrm{M}$ siRNA-156, the TsSP protein expression decreased by $59.98,37.23,36.22$ and $8.71 \%$, respectively, compared with the control siRNA group (Figure 3C). The results indicated that 2.5 $\mu \mathrm{M}$ siRNA-156 had the best knockdown effectiveness on the first day. No obvious

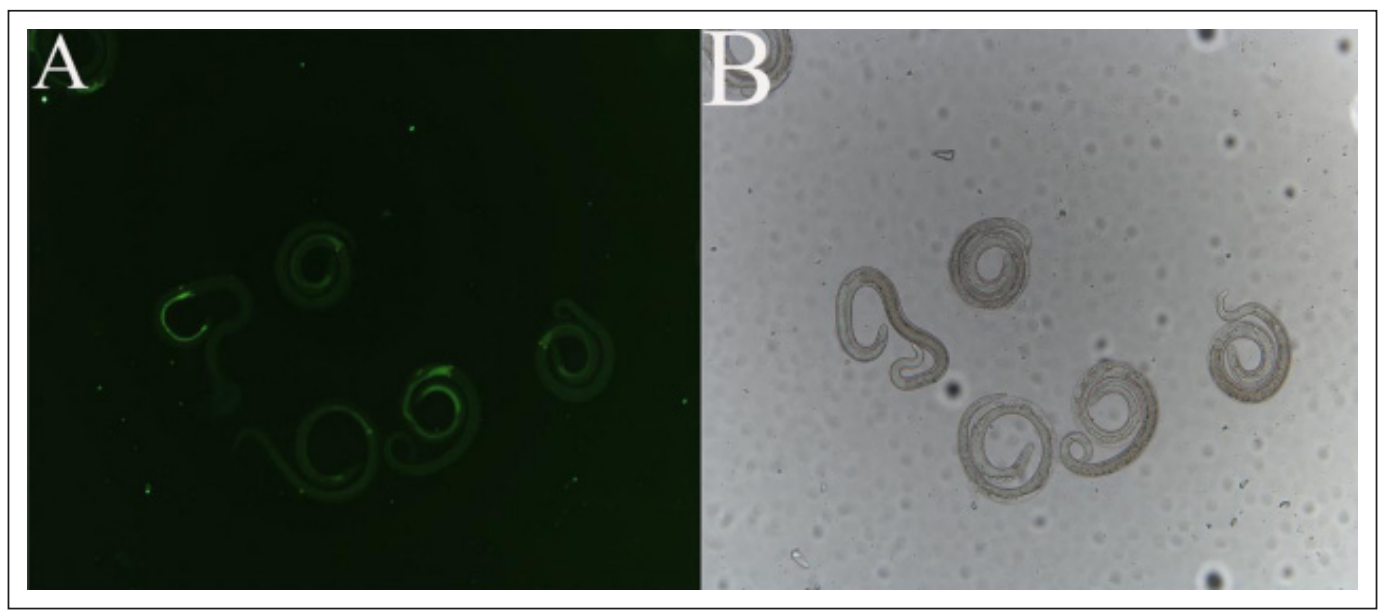

Figure 1. Delivery of FAM-conjugated control siRNA into T. spiralis muscle larvae through electroporation. A: Uptake of siRNA labeled with FAM into the worm after following transfection $(100 \times)$. B: Corresponding white light photo $(100 \times)$ of Figure $1 \mathrm{~A}$. 


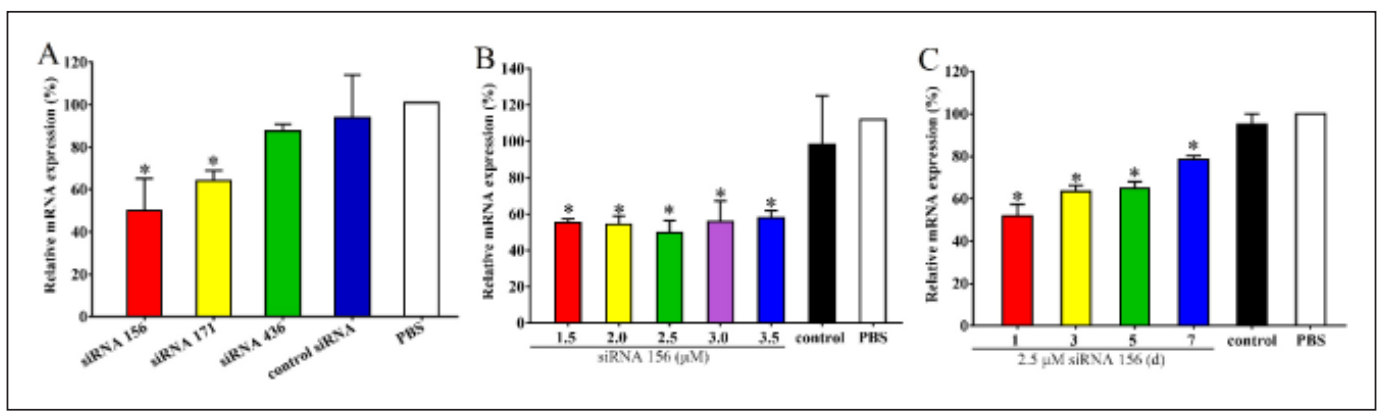

Figure 2. qPCR assay of TsSP mRNA expression level in T. spiralis ML treated with siRNAs. A: Larva TsSP mRNA expression level at 1 day after transfection using diverse siRNAs. B: Larva TsSP mRNA expression level at 1 day after transfection with diverse concentrations of siRNA 156. C: Larva TsSP mRNA expression level at diverse times after transfection with $2.5 \mu \mathrm{M}$ of siRNA 156. Each sample had three repeats, and the results were shown as mean $\pm \mathrm{SD}$. $* P<0.05$ relative to the control siRNA and PBS group.

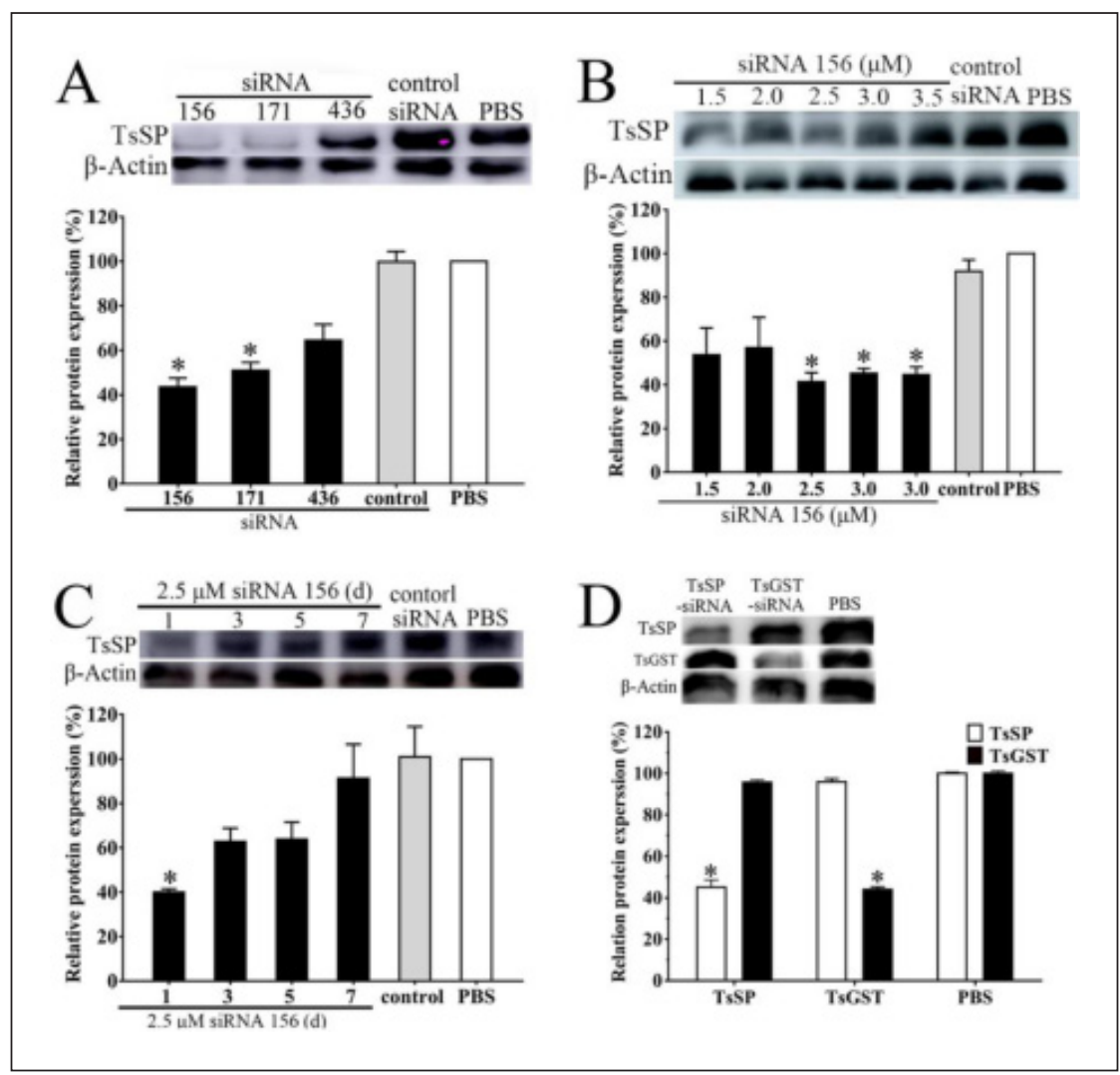

Figure 3. Silencing of TsSP protein expression in T. spiralis larvae following transfection with siRNAs. Western blot results showed that TsSP protein expression in worm crude proteins was suppressed by three TsSP siRNAs (A) at diverse concentrations (B) for various incubation times (C). D: Western blotting of TsSP and TsGST expression level in larvae treated with TsSP siRNA-156 or TsGST siRNA for one day. The relative protein expression level was assessed based on densitometry. Results showed the relative protein expression assessed in three repeated experiment. $* P<0.05$ relative with the control siRNA or PBS group. 
decrease of $\beta$-actin protein expression was seen in muscle larvae transfected using TsSP-siRNA. In the control worm transfected by TsSP-specific siRNA, TsGST protein expression was no suppressed $(P>0.05)$, only TsGST protein expression reduced $57.10 \%$ compared with the PBS group $(P<0.05)$ (Figure 3D), certifying the specificity of TsSP gene silencing mediated by siRNA 156 .

\section{Suppression of TsSP enzymatic activity after siRNA-156 treatment}

The TsSP enzymatic activity following siRNA-156 treatment was detected by degrading azocasein. Among three groups of siRNA-156 treated, control siRNA and PBS control, the serine protease activity of siRNA-156 treated ML ES proteins for degrading azocasein was decreased by $61.38 \%$ relative to the $\mathrm{PBS}$ group $(F=$ $1570.157, P<0.05$ ) (Figure 4 ).

\section{Zymography}

Zymography profiles showed that the somatic crude proteins from the ML treated with siRNA 156, control siRNA and PBS did not hydrolyze the gelatin. However, after the ML was treated with $2.5 \mu \mathrm{M}$ siRNA 156 , the capacity of the ML ES proteins to hydrolyze the gelatin was obviously reduced in comparison to the control siRNA and PBS groups (Figure 5), suggesting that the TsSP exist principally in the ML ES proteins.

\section{No effect of siRNA on larval survival}

When the ML treated with siRNA-156, control siRNA and PBS were cultured at $37^{\circ} \mathrm{C}$ for 1 day, $4.67,4.33$ and $3.33 \%$ of the ML of each group died $\left(\chi^{2}=0.039, P>0.05\right)$. When the treated ML were cultured for 7 days, 33.33, 32.00 and $31.67 \%$ of the ML of each group died $\left(\chi^{2}=0.1000, P>0.05\right)$, indicating that silencing of TsSP expression has no obvious impairment effect on the larval survival.

\section{Inhibition of siRNA-156 on the in vitro larva invasion of IEC}

While the IIL 1 were inoculated onto the IEC monolayer and cultivated for $2 \mathrm{~h}$, the IIL 1 invaded and migrated in the monolayer (Figure 6). The worm invasion rate (40.90\%) of siRNA-156 group was significantly lower

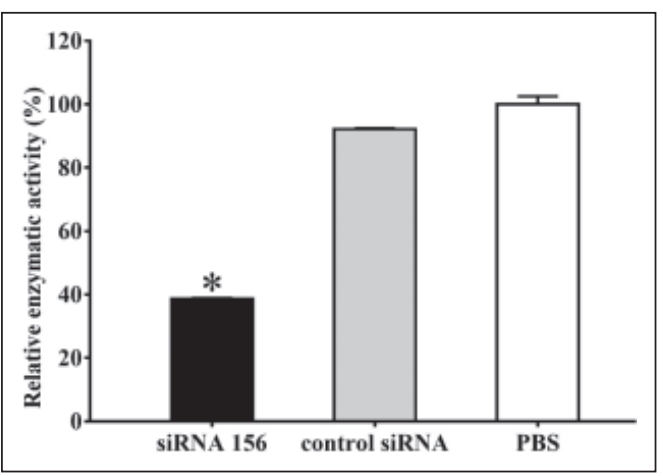

Figure 4. The azocasein degradation by T. spiralis larva ES proteins after siRNA 156 treatment. $* P$ $<0.05$ compared with the control siRNA and PBS group.

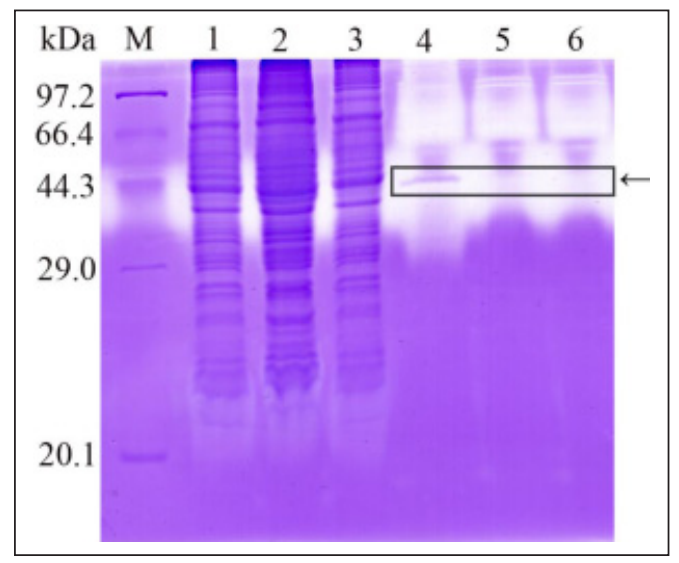

Figure 5. Zymography of T. spiralis serine protease. Lane M: Protein marker; Somatic soluble proteins from the ML treated with siRNA 156 (lane 1), control siRNA (lane 2) and PBS (lane 3) did not hydrolyze the gelatin. However, the capacity of the ML ES proteins from ML treated with siRNA 156 (lane 4) to hydrolyze the gelatin was obviously reduced in comparison to the control siRNA (lane 5) and PBS (lane 6) group, demonstrating the capacity of the ML ES proteins to hydrolyze the gelatin was obviously suppressed after the TsST gene was knockdown using siRNA 156. The arrow represents the protein bands hydrolyzed by the ES proteins from the control siRNA- or PBS-treated ML (lane 5 and 6), but not ES proteins from the siRNA 156-treated ML (lane 4).

than the control siRNA (65.76\%) and PBS group (64.47\%) $\left(\chi^{2}=20.999, P<0.0001\right)$. The results revealed that the knockdown of TsSP gene by siRNA-156 obviously suppressed the IIL1 invasion of IEC. 




Figure 6. Trichinella spiralis IIL1 larvae were inoculated onto IEC monolayer and larva intrusion of IEC was observed by microscopy after incubation for $2 \mathrm{~h}(200 \times)$. A: siRNA 156-treated larva did not invade the IEC monolayer and exhibited the coiled. B: PBS-treated larva invaded the monolayer and was motile. C. Larval invasive rate of each group was presented as mean \pm standard deviation of three independent experiments. ${ }^{*} P<0.05$ compared with the control siRNA and PBS group.

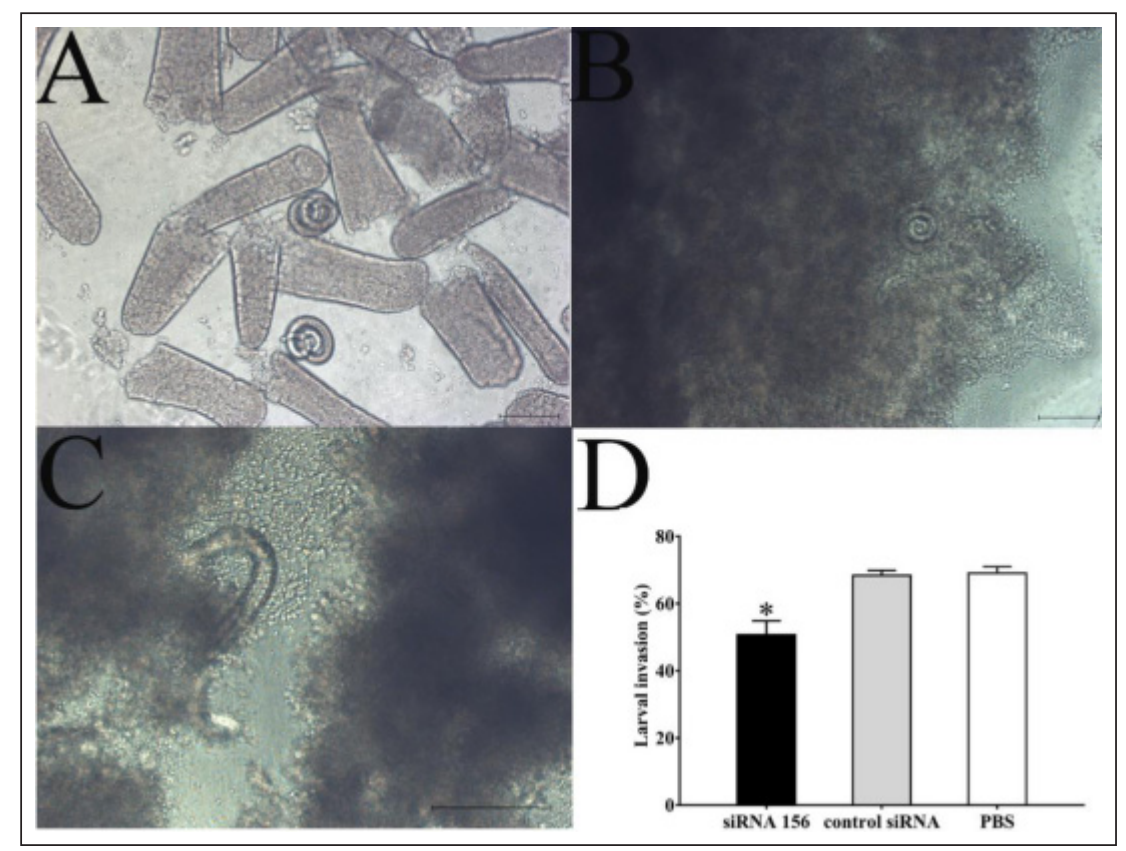

Figure 7. siRNA-156 suppressed on the in vitro larval invasion of enteral mucosa. One hundred IIL1 pretreated with siRNA-156, control siRNA or PBS were injected into the isolated and ligated mouse intestine segment, and cultivated in Tyrode's solution at $37^{\circ} \mathrm{C}, 5 \% \mathrm{CO}_{2}$ for $2 \mathrm{~h}$. The intestine segment was cut off and enteral mucosa were examined on microscopy. The larvae invaded into enteral mucosa were observed and counted. A: siRNA-156 treated larvae did not invade the intestinal mucosa. B: Control siRNA- treated larva invaded into the intestinal mucosa. C: PBS-treated larva invaded into the intestinal mucosa. D: Larva invasion of intestinal mucosa were presented as mean \pm standard deviation of three independent experiments of each group. ${ }^{*} P<0.05$ compared with the PBS group. Scale-bar: $100 \mu \mathrm{m}$.

\section{Suppression of siRNA-156 on the in vivo larval invasion of enteral mucosa}

After being perfused and incubated in the gut for $2 \mathrm{~h}$ with Tyrode's solution, the IIL intruded into intestinal mucosa (Figure 7). The larva invasive rate of siRNA-156, control
siRNA and PBS group was 51.00\%, 68.00\%, and $69.00 \%\left(\chi^{2}=8.748, P<0.05\right)$. The results indicated that siRNA-156 resulted in 26.09\% inhibition of larva invasion of enteral mucosa in comparison to the PBS group. 


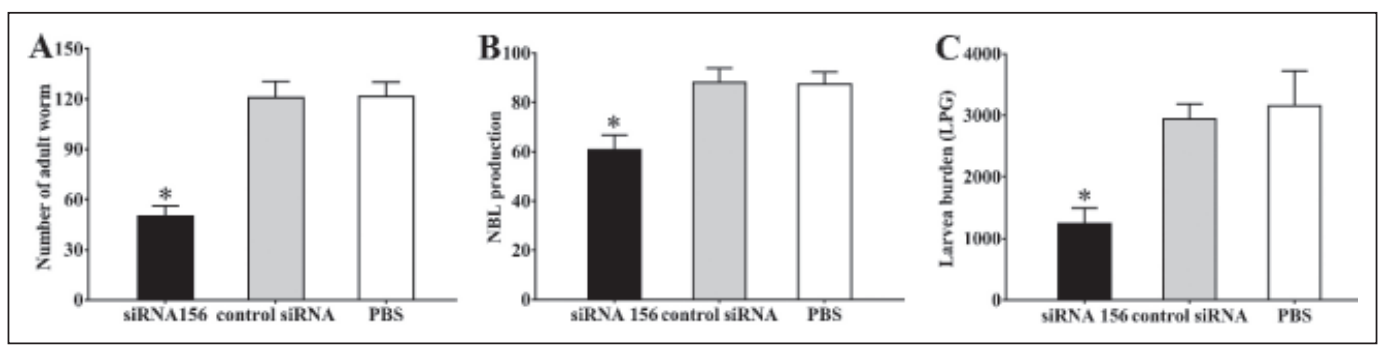

Figure 8. Worm burdens of adults (A) as well as larvae per gram (LPG) of muscles (C), and newborn larvae (NBL) production of females (B) recovered from mice infected with ML electroporated with siRNA-156. The worm burden is shown as mean \pm SD of siRNA-156, control siRNA and PBS group $(\mathrm{n}=10)$. $* P<0.05$ compared with the control siRNA and PBS group.

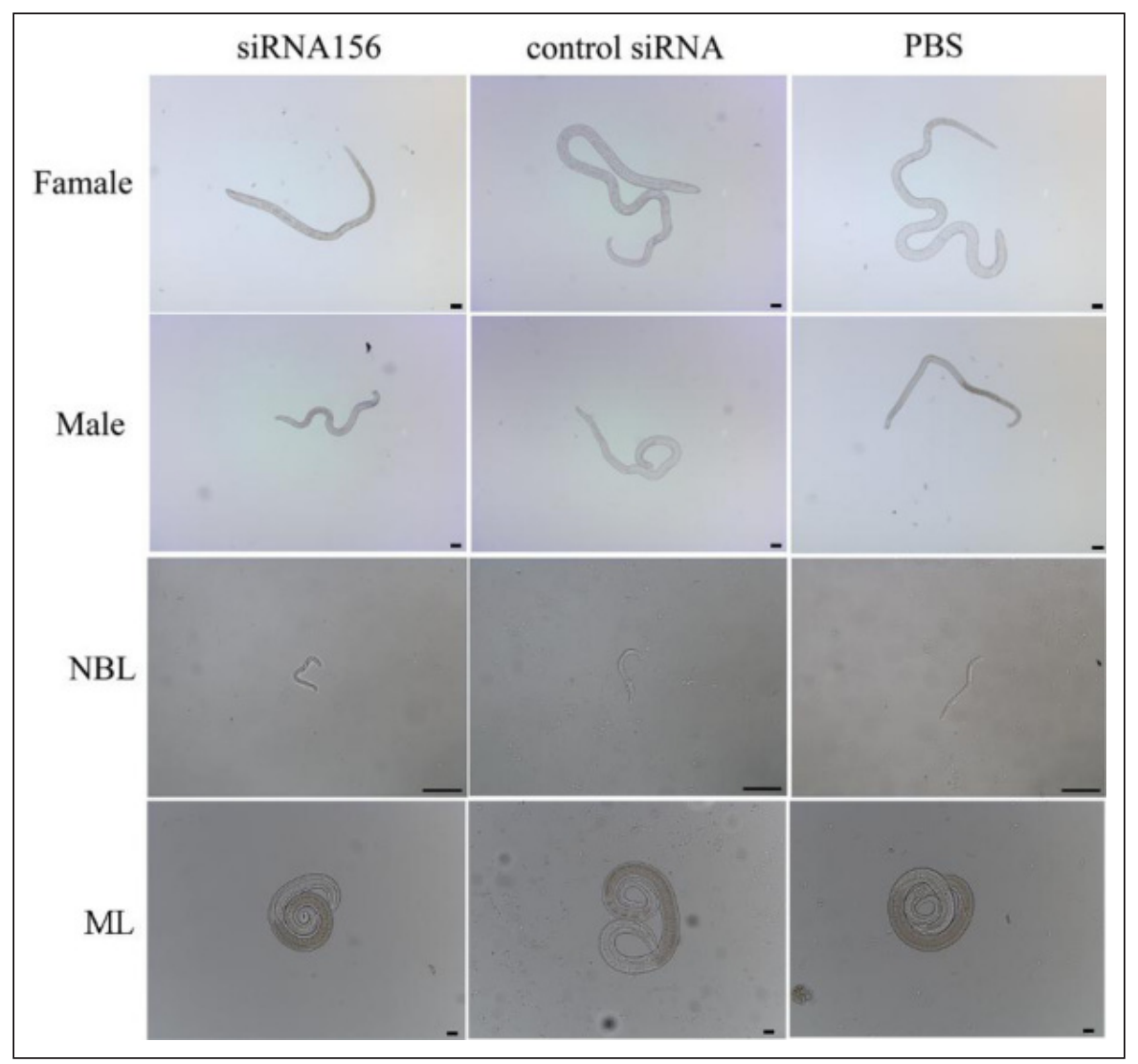

Figure 9. Size of different lifecycle phase worms collected from mice infected with T. spirali ML transfected with siRNA-156. Scale bars of AW and ML = $100 \mu \mathrm{m}$, and scale bars of $\mathrm{NBL}=50 \mu \mathrm{m}$.

Inhibition of siRNA-156 on the in vivo larval infectivity, growth and fecundity Compared to the PBS group, mice infected with ML treated using siRNA-156 exhibited a $58.85 \%$ intestinal AW reduction and $60.48 \%$ ML reduction $\left(F_{\text {adults }}=256.630, F_{\text {larvea }}\right.$ $=81.877, P<0.05)$. There was no obvious AW and ML burden reduction in mice infected by ML treated using the control siRNA (Figure $8 \mathrm{~A}, \mathrm{C})\left(F_{\text {adults }}=0.862, F_{\text {larvea }}\right.$ $=0.262, P>0.05)$.

Moreover, the length of female and male adults recovered from siRNA-156 group was evidently shorter than the control siRNA and PBS groups (Figure 9, 10) $\left(F_{\mathrm{fAW}}=5.817, F_{\mathrm{mAW}}\right.$ $=9.617, P<0.05)$. The in vitro NBL yield 


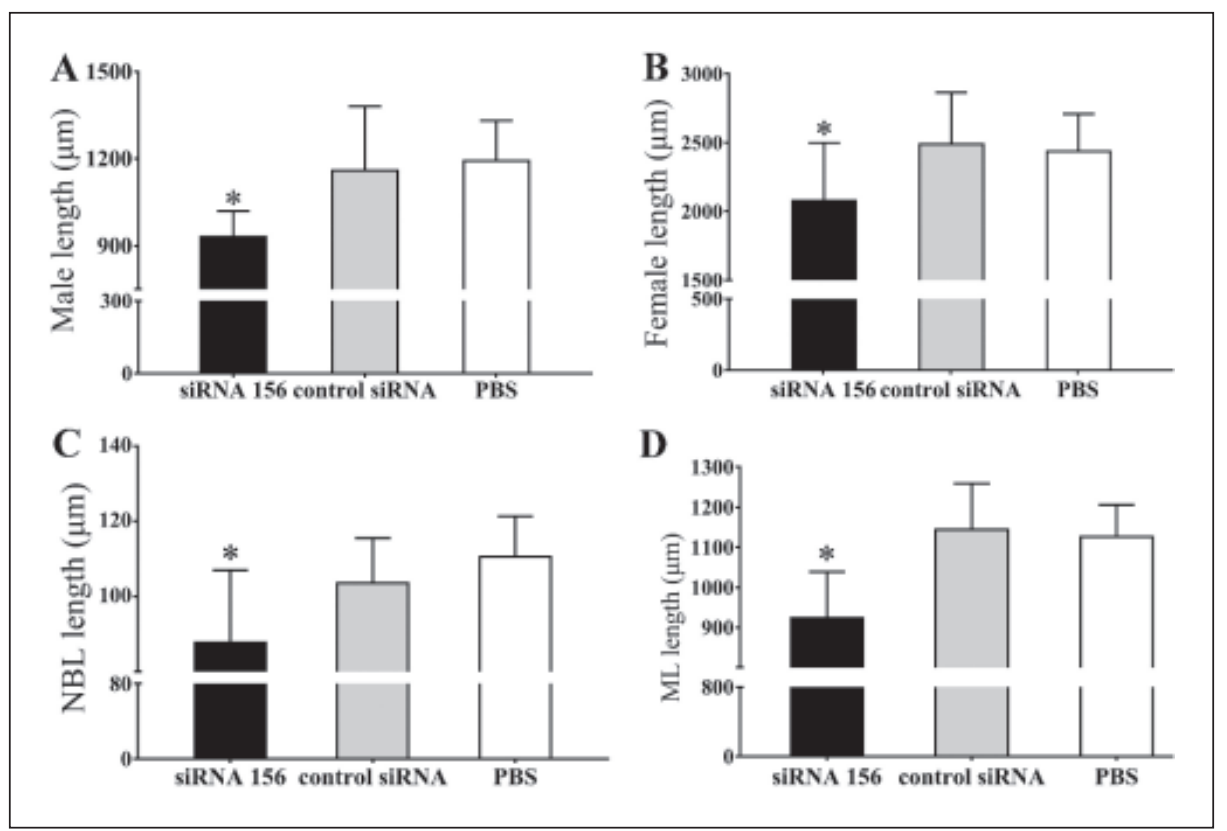

Figure 10. The lengths of diverse phase worms from mice infected with $T$. spiralis larvae transfected using siRNA-156. Ten worms were measured from each mouse. A: Female adults; B: Male adults; C: Newborn larvae (NBL); D: Muscle larvae (ML).

of each adult female of siRNA-156 group was also dramatically decreased relative to the control siRNA or PBS group (Figure 8B) $(F=50.440, P<0.05)$. Additionally, the NBL and ML from siRNA-156 group was notably shorten in comparison to the control siRNA or PBS group (Figure 8, 9) $\left(F_{\mathrm{NBL}}=9.753, F_{\mathrm{ML}}\right.$ $=40.933, P<0.05)$. The results demonstrated that knockdown of TsSP gene by RNAi inhibited the larval infectivity, invasive and growth, and female reproductive ability, and therefore mitigated the Trichinella infection in challenged mice.

\section{DISCUSSION}

The T. spiralis draft genome contains 15808 protein-coding genes (Mitreva et al., 2011), but only a few of T. spiralis gene functions were identified until now (Nagano et al., 2009; Zarlenga et al., 2016). RNAi technique has been widely applied to the function characterization of the nematodes with medical and veterinary importance (e.g., Brugia malayi and Setaria digitate) (Kushwahae et al., 2012; Somarathne et al., 2018). The acts of T. spiralis paratomyosin, nudix hydrolase, and serine protease inhibitor have been also determined through RNAi (Chen et al., 2012; Zhang et al., 2016; Yang et al., 2019). However, the TsSP function in T. spiralis lifecycle is still unknown.

In this study, we used the RNAi silencing method to investigate the TsSP function in lifecycle of $T$. spiralis. On 1 day of transfection with $2.5 \mu \mathrm{M}$ siRNA156, TsSP mRNA and protein expression level was reduced by 48.35 and $59.98 \%$, respectively. Meanwhile, the serine protease activity of siRNA 156 treated ML ES proteins was significantly decreased relative to the control siRNA and PBS group. Previous studies showed that TsSP was located in epi-cuticle and secretory organs of the tissueparasitizing nematode, it was a surface and secreted protein that facilitated the host' enterocyte intrusion by the IIL1 larvae (Sun et al., 2018a, 2018b). TsSP might exerts an important act during worm invasion by means of hydrolyzing the host's extracellular matrix proteins, adhesion proteins and enteral mucins, helping the parasite to break through the native intestinal mucosal barriers, and escaping the immune damage (Dzik, 2006; Hasnain et al., 2012; Toubarro et al., 2010; 
Yang et al., 2015). Our results demonstrated that TsSP-specific siRNA inhibited the expression and enzymatic activity of TsSP.

Additionally, T. spiralis serine protease is a family of proteases which contains eight subfamilies and 81 serine proteases, which own high similarity. The knockdown of one serine protease only suppressed partially the expression and enzymatic activity of TsSP (Yang et al., 2020). Moreover, although the silencing of TsSP expression had no obvious impairment effect on the larval survival, the knockdown of TsST resulted in a significant reduction of the in vitro and in vivo invasive capacity of IIL1 to penetrate into the IEC monolayer and intestinal mucosa.

The results of animal experiment revealed that intestinal adult worm and muscle larval burdens of infected mice with siRNA 156 treated larvae decreased by 58.85 and $60.48 \%$, respectively. When the TsSP gene was knockdown, intestinal worm growth and female reproductive capacity was evidently inhibited, it was demonstrated that adult worms became smaller and the in vitro NBL yield of female adults declined compared with the control siRNA and PBS group. Moreover, other development phase worms (NBL and ML) of siRNA 156-treated group was also prominently smaller than the control groups (as shown in Figure 9 and 10). Previous studies showed that RNAi mediated silencing of some genes from Brugia malayi impaired the embryogenesis, microfilaria release and infective larva development in jirds (Kushwaha et al., 2012). Our results demonstrated that knockdown of TsSP gene by RNAi significantly suppressed the larvae infectivity, invasive and developmental capacity. Additionally, it is evident that that the enteral worm development disability had a direct relation with muscle larva burden reduction in mice challenged using siRNA156-treated larvae, since the uterus length is positively related to the female fecundity index (Murrell et al., 2000; Yang et al., 2019). Our results further verified that TsSP might participate in diverse processes of T. spiralis development, such as intrusion, growth, and reproduction. A further understanding of the TsSP role during a host/nematode interaction might be valuable to screen the new prospective molecular targets of anti-T. spiralis vaccines.

In summary, the results indicated that knockdown of TsSP gene by RNAi significantly reduced the TsSP expression and enzymatic activity, impaired larvae intrusion and growth, and lowered the reproductive capacity of female adults in mice.

\section{Conflicts of interest}

The authors declare no conflicts of interest with regards to this study or the manuscript prepared for publication.

Acknowledgements. This study was supported by grants of the National Natural Science Foundation of China (U1704284; 81971952).

\section{REFERENCES}

Bai, X., Hu, X., Liu, X., Tang, B. \& Liu, M. (2017). Current research of trichinellosis in China. Frontiers in Microbiology 8: 1472.

Chen, X., Yang, Y., Yang, J., Zhang, Z. \& Zhu, X. (2012). RNAi-mediated silencing of paramyosin expression in Trichinella spiralis results in impaired viability of the parasite. PLoS One 7: e49913.

Cui, J., Han, Y., Yue, X., Liu, F., Song, Y.Y., Yan, S.W., Lei, J.J., Zhang, X., Jiang, P. \& Wang, Z.Q. (2019). Vaccination of mice with a recombinant novel cathepsin $\mathrm{B}$ inhibits Trichinella spiralis development, reduces the fecundity and worm burden. Parasites \& Vectors 12: 581.

Cui, J., Jiang, P., Liu, L.N. \& Wang, Z.Q. (2013). Survey of Trichinella infections in domestic pigs from northern and eastern Henan, China. Veterinary Parasitology 194: 133-5.

Cui, J., Ren, H.J., Liu, R.D., Wang, L., Zhang, Z.F. \& Wang, Z.Q. (2013c). Phagedisplayed specific polypeptide antigens induce significant protective immunity against Trichinella spiralis infection in BALB/c mice. Vaccine 31: 1171-1177.

Cui, J., Wang, L., Sun, G.G., Liu, L.N., Zhang, S.B., Liu, R.D., Zhang, X., Jiang, P. \& Wang, Z.Q. (2015b). Characterization of 
a Trichinella spiralis $31 \mathrm{kDa}$ protein and its potential application for the serodiagnosis of trichinellosis. Acta Tropical 142: 57-63.

Cui, J. \& Wang, Z.Q. (2011). An epidemiological overview of swine trichinellosis in China. The Veterinary Journal 190: 324-328.

Cui, J., Wang, Z.Q. \& Xu, B.L. (2011). The epidemiology of human trichinellosis in China during 2004-2009. Acta Tropical 118: $1-5$.

Dzik, J.M. (2006). Molecules released by helminth parasites involved in host colonization. Acta Biochimica Polonica 53: $33-64$.

Food and Agriculture Organization of the United Nations [FAO]/World Health Organization [WHO]. (2014). Multicriteriabased ranking for risk management of food-borne parasites. Microbiological Risk Assessment Series No. 23. Rome: FAO/WHO.

Hasnain, S.Z., McGuckin, M.A., Grencis, R.K. \& Thornton, D.J. (2012). Serine protease (s) secreted by the nematode Trichuris muris degrade the mucus barrier. PLoS Neglected Tropical Diseases 6: e1856.

Jiang, P., Wang, Z.Q., Cui, J. \& Zhang, X. (2012). Comparison of artificial digestion and Baermann's methods for detection of Trichinella spiralis pre-encapsulated larvae in muscles with low-level infections. Foodborne Pathogens and Disease 9: 27-31.

Jiang, P., Zhang, X., Wang, L.A., Han, L.H., Yang, M., Duan, J.Y., Sun, G.G., Qi, X., Liu, R.D., Wang, Z.Q. \& Cui, J. (2016). Survey of Trichinella infection from domestic pigs in the historical endemic areas of Henan province, central China. Parasitology Research 115: 4707-4709.

Kushwaha, S., Singh, P.K., Shahab, M., Pathak, M. \& Bhattacharya, S.M. (2012). In vitro silencing of Brugia malayi trehalose-6phosphate phosphatase impairs embryogenesis and in vivo development of infective larvae in jirds. PLoS Neglected Tropical Diseases 6: e1770.

Li, J.F., Guo, K.X., Qi, X., Lei, J.J., Han, Y., Yan, S.W., Jiang, P., Yu, C., Cheng, X.C., Wang, Z.Q. \& Cui, J. (2018). Protective immunity against Trichinella spiralis in mice elicited by oral vaccination with attenuated Salmonella-delivered TsSP1.2 DNA. Veterinary Research 49: 87.

Li, L.G., Wang, Z.Q., Liu, R.D., Yang, X., Liu, L.N., Sun, G.G., Jiang, P., Zhang, X., Zhang, G.Y. \& Cui, J. (2015). Trichinella spiralis: low vaccine potential of glutathione $\mathrm{S}$ transferase against infections in mice. Acta Tropical 146: 25-32.

Liu, C.Y., Ren, H.N., Song, Y.Y., Sun, G.G., Liu, R.D., Jiang, P., Long, S.R., Zhang, X., Wang, Z.Q. \& Cui, J. (2018). Characterization of a putative glutathione S-transferase of the parasitic nematode Trichinella spiralis. Experimental Parasitology 187: 59-66.

Liu, P., Cui, J., Liu, R.D., Wang, M., Jiang, P., Liu, L.N., Long, S.R., Li, L.G., Zhang, S.B., Zhang, X.Z. \& Wang, Z.Q. (2015a). Protective immunity against Trichinella spiralis infection induced by TsNd vaccine in mice. Parasites \& Vectors 8 : 185.

Liu, R.D., Cui, J., Liu, X.L., Jiang, P., Sun, G.G., Zhang, X., Long, S.R., Wang, L. \& Wang, Z.Q. (2015b). Comparative proteomic analysis of surface proteins of Trichinella spiralis muscle larvae and intestinal infective larvae. Acta Tropical 150: 7986.

Liu, R.D., Jiang, P., Wen, H., Duan, J.Y., Wang, L.A., Li, J.F., Liu, C.Y., Sun, G.G., Wang, Z.Q. \& Cui, J. (2016a). Screening and characterization of early diagnostic antigens in excretory-secretory proteins from Trichinella spiralis intestinal infective larvae by immunoproteomics. Parasitology Research 115: 615-622.

Liu, R.D., Qi, X., Sun, G.G., Jiang, P., Zhang, X., Wang, L.A., Liu, X.L., Wang, Z.Q. \& Cui, J. (2016b). Proteomic analysis of Trichinella spiralis adult worm excretory-secretory proteins recognized by early infection sera. Veterinary Parasitology 231: 43-46.

Liu, R.D., Wang, Z.Q., Wang, L., Long, S.R., Ren, H.J. \& Cui, J. (2013). Analysis of differentially expressed genes of Trichinella spiralis larvae activated by bile and cultured with intestinal epithelial cells using real-time PCR. Parasitology Research 112: 4113-4120. 
Long, S.R., Wang, Z.Q., Jiang, P., Liu, R.D., Qi, X., Liu, P., Ren, H.J., Shi, H.N. \& Cui, J. (2015). Characterization and functional analysis of Trichinella spiralis Nudix hydrolase. Experimental Parasitology 159: 264-273.

Long, Y., Cao, B., Yu, L., Tukayo, M., Feng, C., Wang, Y. \& Luo, D. (2015). Angiostrongylus cantonensis cathepsin B-like protease (Ac-cathB-1) is involved in host gut penetration. Parasite 22: 37.

Lorenzo-Morales, J., Ortega-Rivas, A., Foronda, P., Abreu-Acosta, N., Ballart, D., Martínez, E. \& Valladares, B. (2005). RNA interference (RNAi) for the silencing of extracellular serine proteases genes in Acanthamoeba: molecular analysis and effect on pathogenecity. Molecular and Biochemical Parasitology 144: 10-15.

ManWarren, T., Gagliardo, L., Geyer, J., McVay, C., Pearce-Kelling, S. \& Appleton, J. (1997). Invasion of intestinal epithelia in vitro by the parasitic nematode Trichinella spiralis. Infection and Immunity 65: 4806-4812.

Martinez, J., Perez-Serrano, J., Bernadina, W.E. \& Rodriguez-Caabeiro, F. (2001). Stress response to cold in Trichinella species. Cryobiology 43: 293-302.

McVay, C.S., Tsung, A. \& Appleton, J. (1998). Participation of parasite surface glycoproteins in antibody-mediated protection of epithelial cells against Trichinella spiralis. Infection and Immunity 66: 1941-1945.

Mitreva, M., Jasmer, D.P., Zarlenga, D.S., Wang, Z., Abubucker, S., Martin, J., Taylor, C.M., Yin, Y., Fulton, L., Minx, P., Yang, S.P., Warren, W.C., Fulton, R.S., Bhonagiri, V., Zhang, X., Hallsworth-Pepin, K., Clifton, S.W., McCarter, J.P., Appleton, J., Mardis, E.R. \& Wilson, R.K. (2001). The draft genome of the parasitic nematode Trichinella spiralis. Nature genetics 43: 228-235.

Murrell, K.D., Lichtenfels, R.J., Zarlenga, D.S. \& Pozio, E. (2000). The systematics of the genus Trichinella with a key to species. Veterinary Parasitology 93: 293-307.
Nagano, I., Wu, Z. \& Takahashi, Y. (2009). Functional genes and proteins of Trichinella spp. Parasitology Research 104: 197-207.

Naito, Y., Yoshimura, J., Morishita, S. \& UiTei, K. (2009). siDirect 2.0: updated software for designing functional siRNA with reduced seed-dependent off-target effect. BMC Bioinformatics 10: 392.

Pozio, E. (2007). World distribution of Trichinella spp. infections in animals and humans. Veterinary Parasitology 149: 3-21.

Qi, X., Han, Y., Jiang, P., Yue, X., Ren, H.N., Sun, G.G., Long, S.R., Yu, C., Cheng, X.C., Cui, J. \& Wang, Z.Q. (2018a). Oral vaccination with Trichinella spiralis DNase II DNA vaccine delivered by attenuated Salmonella induces a protective immunity in BALB/c mice. Veterinary Research 49: 119.

Qi, X., Yue, X., Han, Y., Jiang, P., Yang, F., Lei, J.J., Liu, R.D., Zhang, X., Wang, Z.Q. \& Cui, J. (2018b). Characterization of two Trichinella spiralis adult-specific DNase II and their capacity to induce protective immunity. Frontiers in Microbiology 9: 2504 .

Ramírez-Flores, C.J., Cruz-Mirón, R., Arroyo, R., Mondragón-Castelán, M.E., NopalGuerrero, T., González-Pozos, S., RíosCastro, E. \& Mondragón-Flores, R. (2019). Characterization of metalloproteases and serine proteases of Toxoplasma gondiitachyzoites and their effect on epithelial cells. Veterinary Research 118: 289-306.

Ren, H.J., Cui, J., Yang, W., Liu, R.D. \& Wang, Z.Q. (2013). Identification of differentially expressed genes of Trichinella spiralis larvae after exposure to host intestine milieu. PLoS One 8: e67570.

Ren, H.N., Guo, K.X., Zhang, Y., Liu, R.D., Jiang, P., Zhang, X., Wang, L., Cui, J. \& Wang, Z.Q. (2018). Molecular characterization of a $31 \mathrm{kDa}$ protein from Trichinella spiralis and its induced immune protection in BALB/c mice. Parasites \& Vectors 11: 625. 
Robinson, M.W. \& Connolly, B. (2005). Proteomic analysis of the excretorysecretory proteins of the Trichinella spiralis L1 larva, a nematode parasite of skeletal muscle. Proteomics 5: 45254532.

Romaris, F., North, S.J., Gagliardo, L.F., Butcher, B.A., Ghosh, K. \& Beiting, D.P. (2002). A putative serine protease among the excretory-secretory glycoproteins of L1 Trichinella spiralis. Molecular and Biochemical Parasitology 122: 149-160.

Rostami, A., Gamble, H.R., Dupouy-Camet, J., Khazan, H. \& Bruschi, F. (2017). Meat sources of infection for outbreaks of human trichinellosis. Food Microbiology 64: 65-71.

Somarathne, M.B.C.L., Gunawardene, Y.I.N.S., Chandrasekharan, N.V. \& Dassanayake, R.S. (2018). Development of siRNA mediated RNA interference and functional analysis of novel parasitic nematode-specific protein of Setaria digitata. Experimental Parasitology 186: 42-49.

Song, Y.Y., Wang, L.A., Ren, H.N., Qi, X., Sun, G.G., Liu, R.D., Jiang, P., Zhang, X., Cui, J. \& Wang, Z.Q. (2018a). Cloning, expression and characterisation of a cysteine protease from Trichinella spiralis. Folia Parasitologica 65: 007.

Song, Y.Y., Zhang, Y., Ren, H.N., Sun, G.G., Qi, X., Yang, F., Jiang, P., Zhang, X., Cui, J. \& Wang, Z.Q. (2018b). Characterization of a serine protease inhibitor from Trichinella spiralis and its participation in larval invasion of host's intestinal epithelial cells. Parasites \& Vectors 11: 499.

Song, Y.Y., Zhang, Y., Yang, D.Q., Ren, H.N., Sun, G.G., Liu, R.D., Jiang, P., Zhang, X., Cui, J. \& Wang, Z.Q. (2018c). The immune protection induced by a serine protease inhibitor from Trichinella spiralis. Frontiers in Microbiology 9: 1544.

Sun, G.G., Lei, J.J., Guo, K.X., Liu, R.D., Long, S.R., Zhang, X., Jiang, P., Cui, J. \& Wang, Z.Q. (2019a). Primary assessment of a T. spiralis putative serine protease for early serological detection of Trichinella infection. Tropical Biomedicine 36: 792802.

Sun, G.G., Lei, J.J., Ren, H.N., Zhang, Y., Guo, K.X., Long, S.R., Liu, R.D., Jiang, P., Wang, Z.Q. \& Cui, J. (2019b). Intranasal immunization with recombinant Trichinella spiralis serine protease elicits protective immunity in BABL/c mice. Experimental Parasitology 201: $1-10$.

Sun, G.G., Ren, H.N., Liu, R.D., Song, Y.Y., Qi, X., Hu, C.X., Yang, F., Jiang, P., Zhang, X., Wang, Z.Q. \& Cui, J. (2018a). Molecular characterization of a putative serine protease from Trichinella spiralis and its elicited immune protection. Veterinary Research 49: 59.

Sun, G.G., Song, Y.Y., Jiang, P., Ren, H.N., Yan, S.W., Han, Y., Liu, R.D., Zhang, X., Wang, Z.Q. \& Cui, J. (2018b). Characterization of a Trichinella spiralis putative serine protease. Study of its potential as serodiagnostic tool. PLoS Neglected Tropical Diseases 12: e0006485.

Sun, G.G., Wang, Z.Q., Liu, C.Y., Jiang, P., Liu, R.D., Wen, H., Qi, X., Wang, L. \& Cui, J. (2015). Early serodiagnosis of trichinellosis by ELISA using excretorysecretory antigens of Trichinella spiralis adult worms. Parasites \& Vectors 8: 484.

Toubarro, D., Lucena-Robles, M., Nascimento, G., Santos, R., Montiel, R., Veríssimo, P., Pires, E., Faro, C., Coelho, A.V. \& Simões, N. (2010). Serine protease-mediated host invasion by the parasitic nematode Steinernema carpocapsae. The Journal of Biological Chemistry 285: 3066630675.

Wang, B., Wang, Z.Q., Jin, J., Ren, H.J., Liu, L.N. \& Cui, J. (2013c). Cloning, expression and characterization of a Trichinella spiralis serine protease gene encoding a $35.5 \mathrm{kDa}$ protein. Experimental Parasitology 134: 148-154.

Wang, L., Cui, J., Hu, D.D., Liu, R.D. \& Wang, Z.Q. (2014). Identification of early diagnostic antigens from major excretorysecretory proteins of Trichinella spiralis muscle larvae using immunoproteomics. Parasites \& Vectors 7: 40. 
Wang, L., Wang, Z.Q. \& Cui, J. (2013a). Protein changes in Trichinella spiralis muscle larvae in vitro induced by bovine bile. Veterinary Parasitology 194: 164-167.

Wang, L., Wang, Z.Q., Hu, D.D. \& Cui, J. (2013b). Proteomic analysis of Trichinella spiralis muscle larval excretorysecretory proteins recognized by early infection sera. BioMed Research International 2013: 139745.

Wang, S.W., Wang, Z.Q. \& Cui, J. (2011). Protein change of intestinal epithelial cells induced in vitro by Trichinella spiralis infective larvae. Parasitology Research 108: 593-599.

Wang, Z.Q., Li, L.Z., Jiang, P., Liu, L.N. \& Cui, J. (2012a). Molecular identification and phylogenetic analysis of Trichinella isolates from different provinces in mainland China. Parasitology Research 110: $753-757$.

Wang, Z.Q., Liu, R.D., Sun, G.G., Song, Y.Y., Jiang, P., Zhang, X. \& Cui, J. (2017). Proteomic analysis of Trichinella spiralis adult worm excretory-secretory proteins recognized by sera of patients with early trichinellosis. Frontiers in Microbiology 8: 986.

Wang, Z.Q., Wang, L. \& Cui, J. (2012b). Proteomic analysis of Trichinella spiralis proteins in intestinal epithelial cells after culture with their larvae by shotgun LC-MS/MS approach. Journal of Proteomics 75: 2375-2383.

Wang, Z.Q., Zhang, S.B., Jiang, P., Liu, R.D., Long, S.R., Zhang, X., Ren, H.J. \& Cui, J. (2015). The siRNA-mediated silencing of Trichinella spiralis Nudix hydrolase results in reduction of larval infectivity. Parasitology Research 114: 3551-3557.

$\mathrm{Xu}$, J., Liu, R.D., Long, S.R., Jiang, P., Zhang, X., Cui, J. \& Wang, Z.Q. (2020). Characterization of a chymotrypsin-like enzyme from Trichinella spiralis and its facilitation on larva penetration of host's enteral epithelial cells. Research in Veterinary Science 128: 1-8.
Xu, J., Yang, F., Yang, D.Q., Jiang, P., Liu R.D., Zhang, X., Cui, J. \& Wang, Z.Q. (2018). Molecular characterization of Trichinella spiralis galectin and its participation in larval invasion of host's intestinal epithelial cells. Veterinary Research 49: 79 .

Yang, D.Q., Liu, F., Bai, Y., Zeng, J., Hao, H.N., Yue, X., Hu, C.X., Long, S.R., Liu, R.D., Wang, Z.Q. \& Cui, J. (2020). Functional characterization of glutathione $\mathrm{S}$ transferase in Trichinella spiralis invasion, development and reproduction. Veternary Parasitology doi: https://doi.org/10.1016/ j.vetpar.

Yang, F., Yang, D.Q., Song, Y.Y., Guo, K.X., Li, Y.L., Long, S.R., Jiang, P., Cui, J. \& Wang, Z.Q. (2019). In vitro silencing of a serine protease inhibitor suppresses Trichinella spiralis invasion, development, and fecundity. Parasitology Research 118 : 2247-2255.

Yang, F., Guo, K.X., Yang, D.Q., Liu, R.D., Long, S.R., Zhang, X., Jiang, P., Cui, J., Wang, Z.Q. (2020). Functional analysis of Trichinella spiralis serine protease 1.2 by siRNA mediated RNA interference. Tropical Biomedicine 37: 1-13.

Yang, Y., Wen, Y.J., Cai, Y.N., Vallée, I., Boireau, P., Liu, M.Y. \& Cheng, S.P. (2015). Serine proteases of parasitic helminths. The Korean Journal of Parasitology 53: 111.

Zarlenga, D., Wang, Z. \& Mitreva, M. (2016). Trichinella spiralis: Adaptation and parasitism. Veternary Parasitology 231: 8-21.

Zhang, S.B., Jiang, P., Wang, Z.Q., Long, S.R. Liu, R.D., Zhang, X., Yang, W., Ren, H.J. \& Cui, J. (2016). DsRNA-mediated silencing of Nudix hydrolase in Trichinella spiralis inhibits the larval invasion and survival in mice. Experimental Parasitology 162 : $35-42$.

Zhang, Y.L., Wang, Z.Q., Li, L.G. \& Cui, J. (2013). Molecular characterization of Trichinella spiralis aminopeptidase and its potential as a novel vaccine candidate antigen against trichinellosis in BALB/c mice. Parasites \& Vectors 6: 246. 